ARTICLE

\title{
Self-filtering narrowband high performance organic photodetectors enabled by manipulating localized Frenkel exciton dissociation
}

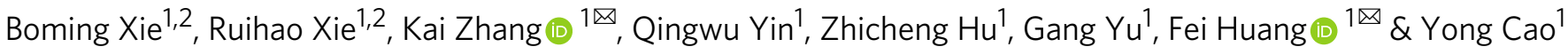

The high binding energy and low diffusion length of photogenerated Frenkel excitons have long been viewed as major drawbacks of organic semiconductors. Therefore, bulk heterojunction structure has been widely adopted to assist exciton dissociation in organic photonelectron conversion devices. Here, we demonstrate that these intrinsically "poor" properties of Frenkel excitons, in fact, offer great opportunities to achieve self-filtering narrowband organic photodetectors with the help of a hierarchical device structure to intentionally manipulate the dissociation of Frenkel excitons. With this strategy, filter-free narrowband organic photodetector centered at $860 \mathrm{~nm}$ with full-width-at-half-maximum of around $50 \mathrm{~nm}$, peak external quantum efficiency around $65 \%$ and peak specific detectivity over $10^{13}$ Jones are obtained, which is one the best performed no-gain type narrowband organic photodetectors ever reported and comparable to commercialized silicon photodetectors. This novel device structure along with its design concept may help create low cost and reliable narrowband organic photodetectors for practical applications.

\footnotetext{
${ }^{1}$ State Key Laboratory of Luminescent Materials and Devices, Institute of Polymer Optoelectronic Materials and Devices, School of Materials Science and Engineering, South China University of Technology, 381 Wushan Road, 510640 Guangzhou, China. ${ }^{2}$ These authors contributed equally: Boming Xie, Ruihao Xie. ${ }^{\circledR}$ email: mszhangk@scut.edu.cn; msfhuang@scut.edu.cn
} 


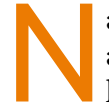
arrowband photodetectors, which only detect light within a specific wavelength of interest and have no response to light of other wavelengths (usually background or environmental radiation), have been widely used in imaging, chemical analysis, and have also shown great potential for application in emerging artificial intelligence networks, such as augmented/virtual reality, advanced driver assistance systems and full-weather robots ${ }^{1-3}$. Commercially available narrowband photodetectors are made of inorganic semiconductors (mostly silicon) and integrated with optical filters ${ }^{4}$. The use of filter creates additional optical interfaces, increases the device's architectural complexity and limits the pixel density of the detector array ${ }^{5}$. Moreover, due to the small extinction coefficient of silicon, a thick silicon film $(\sim 3 \mu \mathrm{m})$ is often required to sufficiently absorb light for operation, which results in an array with a greater likelihood of interpixel cross-talk. To overcome these defects, several strategies are emerging for narrowband photodetection without filter, including: (1) using absorbers with narrowband absorption ${ }^{6,7} ;(2)$ intentionally enhancing the absorption in a selected wavelength range by the plasmonic effect ${ }^{8-10}$; and (3) manipulating the internal quantum efficiency via charge collection narrowing $(\mathrm{CCN})^{11-13}$. These pioneer works provided effective strategies of making filter-free narrowband photodetectors. Nevertheless, in order to suppress the response of the background light and realize the narrowband characteristic, most of these methods have to sacrifice the original sensitivity of the photodetectors and the resulting devices' performances are still behind those of silicon photodetectors.

Among all kinds of semiconductors, organic semiconductors have high extinction coefficients and can efficiently absorb light at thickness as little as a few hundred nanometers. The absorption spectrum of organic semiconductors can be easily tuned by adjusting their molecular structures ${ }^{14}$. In addition, because organic semiconductors are light-weight, flexible, and soluble in organic solvents, they are more suitable for wide applications that require inexpensive, flexible, and moldable photodetectors ${ }^{15-18}$. However, the typical high binding energy and short diffusion length of the excitons in organic semiconductors bring some challenges for their applications in photon-electron conversion devices. Unlike its inorganic counterparts, when an organic semiconductor absorbs a photon, a localized Frenkel exciton is created due to the weak intermolecular van der Waals interaction alongside with the low dielectric constant ${ }^{19,20}$. This exciton cannot spontaneously separate into free charge due to the strong Coulombic interaction ${ }^{21}$, and the short lifespan of exciton limits its diffusion length within tens of nanometers ${ }^{22}$. Therefore, many strategies, including molecular structure modification to improve dielectric constant ${ }^{23-25}$, and matching materials with different electron affinities (ionization potentials) to provide donor-acceptor (D/A) interfaces ${ }^{26,27}$, have been adopted to assist exciton dissociation before dissipation in organic photon-electron conversion devices.

Herein, we find that these intrinsic "poor" properties of Frenkel excitons actually offer organic semiconductors great opportunities to achieve filter-free narrowband organic photodetectors (OPDs). We propose a simple strategy to produce narrowband OPDs by manipulating exciton dissociation (named as "exciton dissociation narrowing" [EDN]), with a hierarchical device structure where thick larger bandgap donor layers followed by a lower bandgap acceptor layer. During the operation, excitons generated by high-energy photons in donor front layers fail to separate into free charges due to the absence of D/A interfaces, thus dissipated. Only low-energy photons with a long penetration depth can reach the D/A interfaces and produce free charges for collection. Some other strategies based on thick active layer with bulk heterojunction $(\mathrm{BHJ})$ structure have also been proposed to construct narrowband photodetectors, including no-gain type $\mathrm{e}^{11}$ and gain type ${ }^{28,29}$. For example, the no-gain type narrowband detectors enabled by $\mathrm{CCN}$ concept modulate the external quantum efficiency (EQE) spectrum by manipulating the collection efficiency of free charges at the corresponding electrodes (photogenerated excitons firstly dissociate into free charges). The gain type detectors achieve photomultiplication via charge tunneling injection from external circuit under large applied voltage. In comparison, the novel device structure and its generic methodology proposed in this study is based on hierarchical active-layer structure and novel working mechanism of EDN. It was interestingly found that this novel methodology can efficiently suppress the response outside the detection window while retaining high sensitivity in the detection region, which enabled us to produce a series of simple structure visible-blind near-infrared (NIR) narrowband OPDs with outstanding performances.

\section{Results}

Basic device structure of self-filtering narrowband OPD. Figure 1a shows the chemical structures of the materials $\mathrm{s}^{30-33}$ used in this study. NT812 is a home designed high charge mobility $\left(\sim 10^{-2} \mathrm{~cm}^{2} \mathrm{~V}^{-1} \mathrm{~s}^{-1}\right)$ donor polymer which shows ideal charge transport in organic solar cells even when the thickness reach to $1 \mu \mathrm{m}^{30,34}$. The most crucial part of the proposed selffiltering (SF) narrowband OPD is the hierarchical structure of a thick larger bandgap donor layer followed by a lower bandgap acceptor layer. This hierarchical structure is usually difficult to be realized because the subsequent acceptor material will penetrate into the underlying donor layer network during solution processing ${ }^{35,36}$. To this end, a combination of methods was adopted. Firstly, to prevent the diffusion of the acceptor molecules (Y6, Fig. 1a), the donor polymer NT812 was crosslinked to be a robust film (with a thickness of $650 \mathrm{~nm}$ ) by the azide crosslinker s-4PFA, which can crosslink semiconducting polymers with a sufficiently low concentration and has negligible influence on their crucial semiconductor properties ${ }^{37}$. Following that, a thin film of NT812 (100 nm) was deposited onto crosslinked NT812 to provide an interdiffusion region with following Y6 for efficient charge separation ${ }^{38}$. Then Y6 was deposited onto NT812 as the rear-layer to obtain a complete activelayer with a total thickness of $800 \mathrm{~nm}$. Here, chloroform was selected as the solvent for rearlayer processing, in which Y6 exhibits good solubility, whereas the front-layer NT812 is nearly insoluble (as shown in Supplementary Fig. 1). This orthogonal solvent and its low boiling point further help the construction of the hierarchical structure.

To verify the existence of this hierarchical structure, we carried out the time-of-flight secondary ion mass spectrometry (ToFSIMS) depth profiling measurement ${ }^{39}$. The $\mathrm{C}_{74} \mathrm{H}_{69} \mathrm{~F}_{4} \mathrm{~N}_{8} \mathrm{O}_{2} \mathrm{~S}_{5}{ }^{-}$ (fragment structure is shown in Supplementary Fig. 2) and the $\mathrm{SN}^{-}$ions were selected to track the depth distribution of $\mathrm{Y} 6$ and NT812, respectively. Figure $1 \mathrm{~b}$ shows the ToF-SIMS depth profile through the prepared film. As expected, Y6 signal shows strong intensity at the first $130 \mathrm{~nm}$, and begins to fall after $130 \mathrm{~nm}$ and tends to be zero after $168 \mathrm{~nm}$. Meanwhile, the intensity of $\mathrm{SN}^{-}$ion begins to rise after $130 \mathrm{~nm}$ and stabilize after $168 \mathrm{~nm}$, which we speculate it is due to the higher thiadiazole content within NT812 leading to higher $\mathrm{SN}^{-}$ion yield. Figure 1c shows the reconstructed three-dimensional chemical images of the $\mathrm{C}_{74} \mathrm{H}_{69} \mathrm{~F}_{4} \mathrm{~N}_{8} \mathrm{O}_{2} \mathrm{~S}_{5}{ }^{-}$and $\mathrm{SN}^{-}$ions, which exhibit evidently that Y6 molecules are concentrated in the upper layer of the whole film with a penetration depth of $168 \mathrm{~nm}$. These results demonstrate that using the methods described above, we effectively restricted the diffusion of Y6 molecules into the underlying donor layer, guarantee a complete device structure of ITO/PEDOT:PSS/donor front layer NT812/ acceptor rear layer $\mathrm{Y6} / \mathrm{PFN}-\mathrm{Br} / \mathrm{Ag}$ (as illustrated in Fig. 1d). 
a
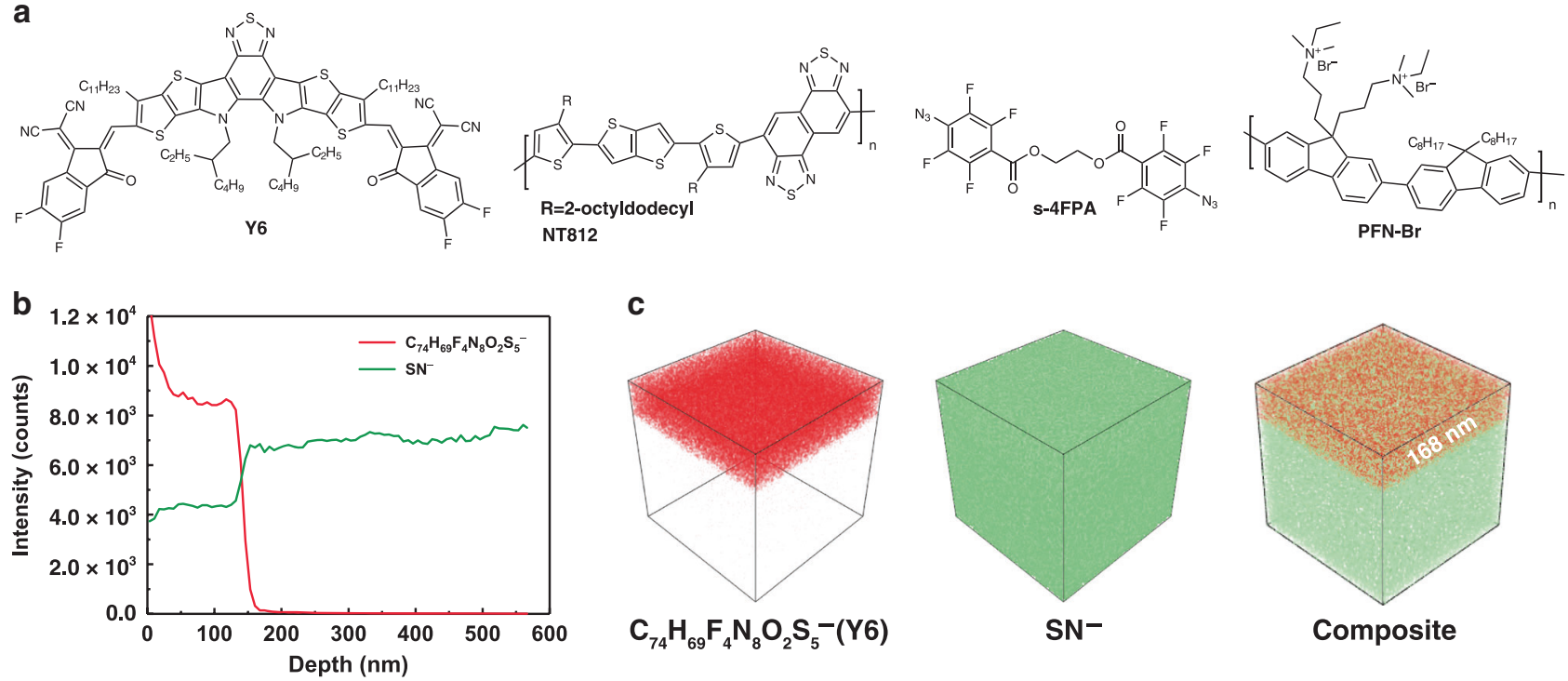

C
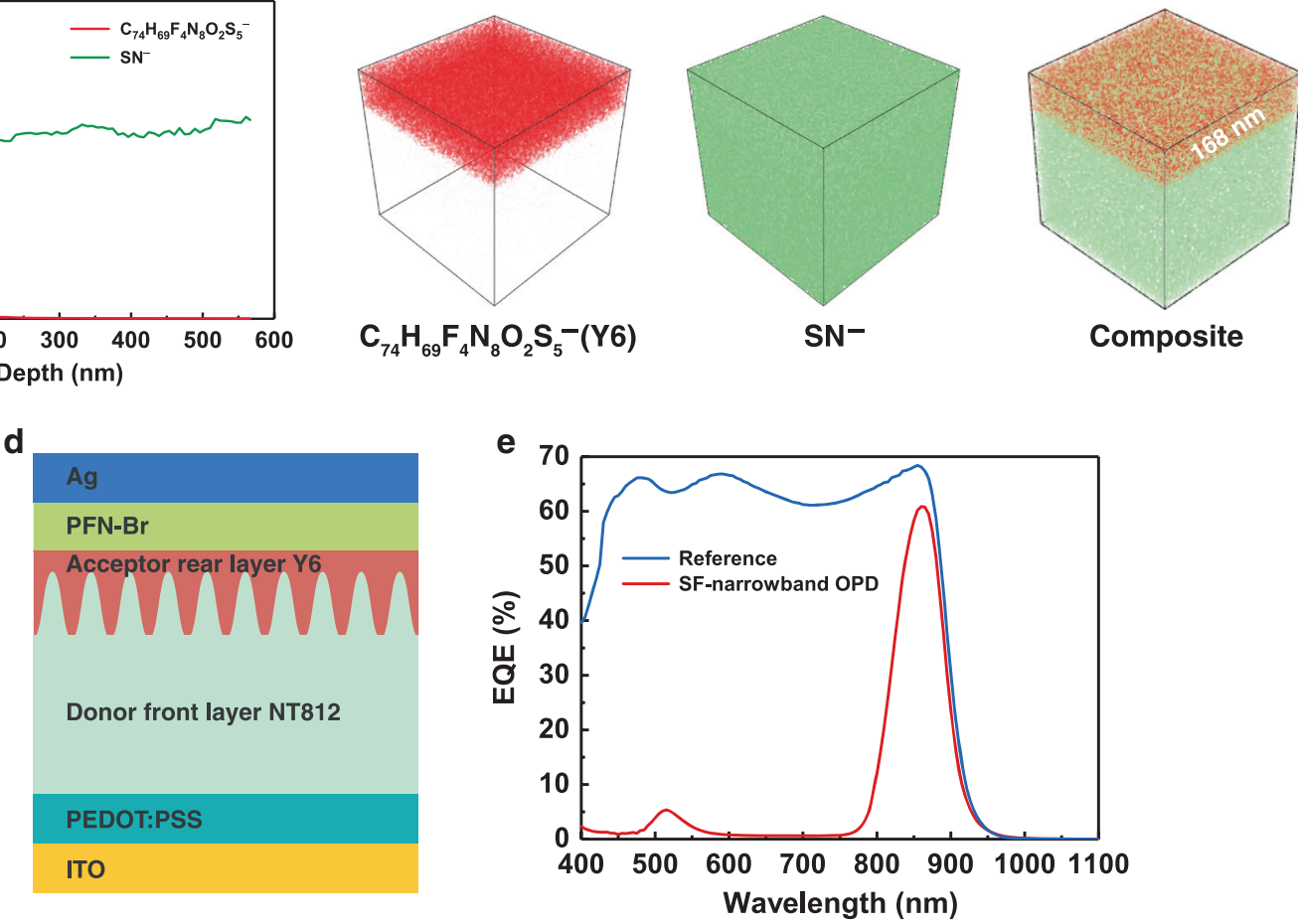

Fig. 1 Hierarchical structure and EQE response of OPDs. a Chemical structures of Y6, NT812, s-4FPA and PFN-Br. b Time-of-flight secondary ion mass spectrometry (ToF-SIMS) depth profile through the prepared film. c Reconstructed three-dimensional negative ion images (100 $\mu \mathrm{m} \times 100 \mu \mathrm{m}$ area) of $\mathrm{C}_{74} \mathrm{H}_{69} \mathrm{~F}_{4} \mathrm{~N}_{8} \mathrm{O}_{2} \mathrm{~S}_{5}{ }^{-}, \mathrm{SN}^{-}$and their composite from a depth profile of the prepared film. $\mathbf{d}$ Basic device structure of self-filtering (SF) narrowband organic photodetector (OPD). e External quantum efficiency (EQE) curves of the SF-narrowband OPD and reference device under - $0.1 \mathrm{~V}$ bias.

Figure 1e presents the EQE spectrum of the fabricated photodetector under $-0.1 \mathrm{~V}$ bias, which exhibits a dominant narrow peak at $860 \mathrm{~nm}$ accompanied by a weak response at $520 \mathrm{~nm}$. Figure 1e also gives the EQE spectrum of the reference device with a conventional structure (ITO/PEDOT:PSS/NT812: Y6 $(150 \mathrm{~nm}) / \mathrm{PFN}-\mathrm{Br} / \mathrm{Ag})$. EQE curves of the SF-narrowband OPD and the reference device show that, as the corresponding $\mathrm{EQE}$ response in visible spectrum is effectively suppressed in SFnarrowband OPD, the peak EQE at $860 \mathrm{~nm}$ is not significantly reduced, maintaining more than $89 \%$ of the corresponding value of reference device. By comparison, the $\mathrm{BHJ}$ device ${ }^{11}$ with device struture of ITO/PEDOT:PSS/NT812:Y6 (800 nm, 1:4)/PFN-Br/ Ag suffers tremendous EQE loss, only obtains a peak EQE around 10\% (as demonstrated in Supplementary Fig. 3).

Working mechanism of self-filtering narrowband OPDs. To understand the mechanism of EDN, the distribution of photogenerated excitons in the bulk of the front-layer material NT812 was analyzed. The continuity equation for neutral excitons can be described by Eq. (1):

$$
\frac{\partial n(x, t)}{\partial t}=g \alpha N_{0}(t) e^{-\alpha x}-\frac{n(x, t)}{\tau}+D \frac{\partial^{2} n(x, t)}{\partial x^{2}}-F\left(x-x_{\text {int }}\right) n(x, t),
$$

where $n(x, t)$ is the time-dependent exciton density, $x$ is the penetration depth of the incident light from the transparent electrode in the bulk of the front layer, $g$ is the internal efficiency of photon-to-exciton, $N_{0}(t)$ is the number of incident photons, $\alpha$ is the absorption coefficient of the front layer material, $\tau$ is the exciton lifetime, $D$ is the exciton diffusion coefficient, and $F\left(x-x_{\text {int }}\right)$ is the exciton dissociation rate at the D/A interface $\left(x_{\text {int }}\right)$. Equation (1) is solved with the stationary illumination $((\partial n / \partial t)=0)$ and boundary conditions prosed by Stübinger et al. ${ }^{40}$.

$$
n(x)=\frac{g N_{0}}{D} \frac{\alpha L^{2}}{1-(\alpha L)^{2}}\left(e^{-\alpha x}-e^{-(x / L)}\right)
$$

where $L$ is the one-dimensional diffusion length, which is defined as $L=\sqrt{D \tau}$. Equation (2) offers the exciton density distribution in the bulk of the donor front layer. Furthermore, the exciton diffusion coefficient $D$ of the front-layer material NT812 is calculated by using the Monte Carlo simulation method ${ }^{41}$. (The specific simulation details are recorded in Supplementary Note 1). Figure 2a shows the experimentally measured time-resolved photoluminescence of the pristine and blend film with a $\mathrm{PC}_{61} \mathrm{BM}$ volume fraction of $0.05 \%$ for NT812 (open circles), and the photoluminescence decay of blend film, which was modeled with the Monte Carlo simulation, is also depicted as solid lines. The model fits the experimental data very well and yields the initial diffusion coefficient $D$ of $19.5 \times 10^{4} \mathrm{~cm}^{2} \mathrm{~s}^{-1}$; the diffusion length 

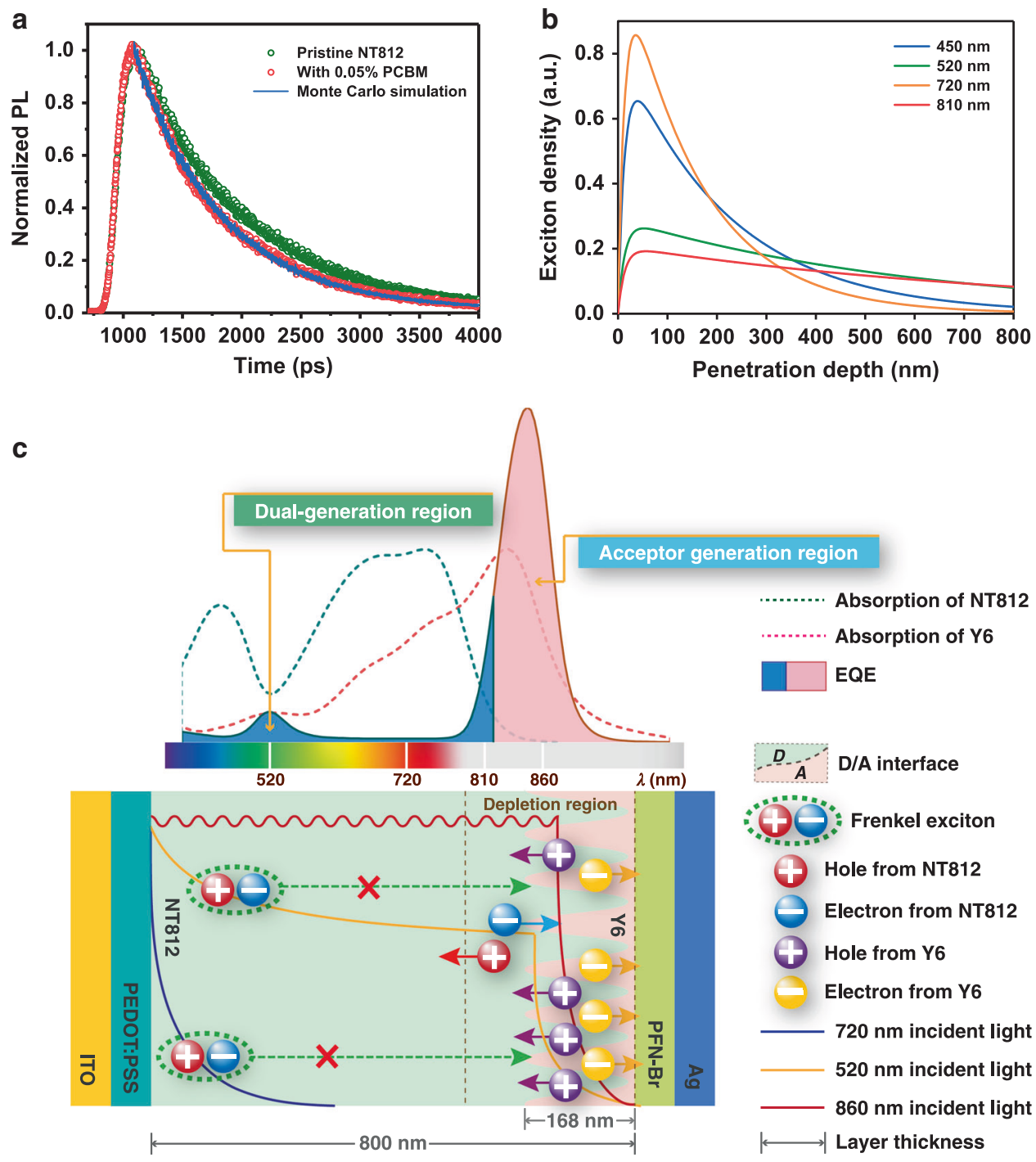

Fig. 2 Working mechanism of SF-narrowband photodetectors. a Experimentally measured time-resolved photoluminescence of pristine (green open circles) and blend film (red open circles). Photoluminescence decay modeled with the Monte Carlo simulation of blend film (blue solid lines). b Exciton density in the bulk of NT812 versus penetration depth of incident light with different wavelength from the transparent ITO electrode. c Diagrammatic illustration of the working mechanism of self-filtering (SF) narrowband photodetectors.

$L$ was then calculated as $13.3 \mathrm{~nm}$. Combined with the value of $\alpha$ measured from the ultraviolet-visible (UV-vis) light absorption spectrum (Supplementary Fig. 4), the exciton density distribution curves of incident light of various wavelengths can be eventually obtained (Fig. 2b). From the distribution of excitons in the donor front layer, the working mechanism of the SF-narrowband OPD can be summarized as follows (as illustrated in Fig. 2c).

For incident light within the absorption range of the front donor material (i.e., high-energy photons in the spectral range of $380-850 \mathrm{~nm}$ for NT812), the corresponding photogenerated excitons are concentrated mainly on the front side of the film (e.g., within the first $200 \mathrm{~nm}$ layer, as shown in Fig. 2b), where the distance between these excitons and the D/A interfaces is far beyond the exciton diffusion length. During their limited lifetime, most excitons outside the depletion region cannot diffuse to the $\mathrm{D} / \mathrm{A}$ interface to dissociate into free charges; therefore, the excitons generated by this spectral range of incident photons are bound in the bulk of the front donor material and eventually relax back to the ground state ${ }^{42,43}$. Moreover, according to the Beer-Lambert law ${ }^{44}$, the intensity of incident light decreases exponentially with the penetration depth in the front layer. Thus, most of the high-energy incident photons were absorbed by the thick front layer and cannot reach the acceptor rear layer (as shown by transmittance spectrum in Supplementary Fig. 5). As a result, neither the donor nor the acceptor material can contribute to the EQE in this spectral range of incident light. (as is typically represented by $720 \mathrm{~nm}$ incident light in Fig. 2b, c).

For incident light with a longer wavelength (longer than the absorption onset of the front donor material; i.e., $\lambda>850 \mathrm{~nm}$ for NT812), the corresponding low-energy photons can penetrate the entire front layer and be harvested by the rear acceptor material with tiny loss. The excitons generated in the acceptor material then efficiently dissociate and separate into free charges at the D/ A interfaces and are collected by the corresponding electrodes, so the corresponding EQE response is obtained at the long wavelength spectrum, which we call the acceptor generation region, which is the main contribution to the narrow response peak. (As that of $860 \mathrm{~nm}$ incident light in Fig. 2c).

However, organic optoelectronic materials generally do not exhibit uniform absorption coefficients over a wide spectral range, photons within a specific spectral region (e.g., 520 and $810 \mathrm{~nm}$ for NT812; see Supplementary Fig. 4) can still penetrate deeply into the 
bulk of the donor front layer and create a small number of excitons within the depletion region (as shown in Fig. 2b). This portion of the incident photons can also partially reach the acceptor rear layer and create excitons for dissociation due to the low absorption coefficient of the front donor material (see Supplementary Fig. 5). Thus, the weak EQE response that corresponds to these incident spectral ranges can be attributed to both the donor and acceptor layer, which we call the dual-generation region.

Self-filtering narrowband OPD with double donor layers. To further suppress the EQE response within the dual-generation region (spectra range of 500-600 nm), poly(3-hexylthiophene) $(\mathrm{P} 3 \mathrm{HT})^{45}$, which provides a cascade highest occupied molecular orbital (HOMO) level with NT812 (see Fig. 3a), was selected to replace PEDOT:PSS as the SF hole transport layer (SF-HTL). P3HT was also crosslinked by s-4FPA to prevent it from being washed away by the following solution, as verified by the crosssection scanning electron microscope image (Fig. 3b). The double donor layers that containing NT812 and P3HT delivered a complete device structure of ITO/SF-HTL P3HT/donor front layer NT812/acceptor rear layer Y6/PFN-Br/Ag.
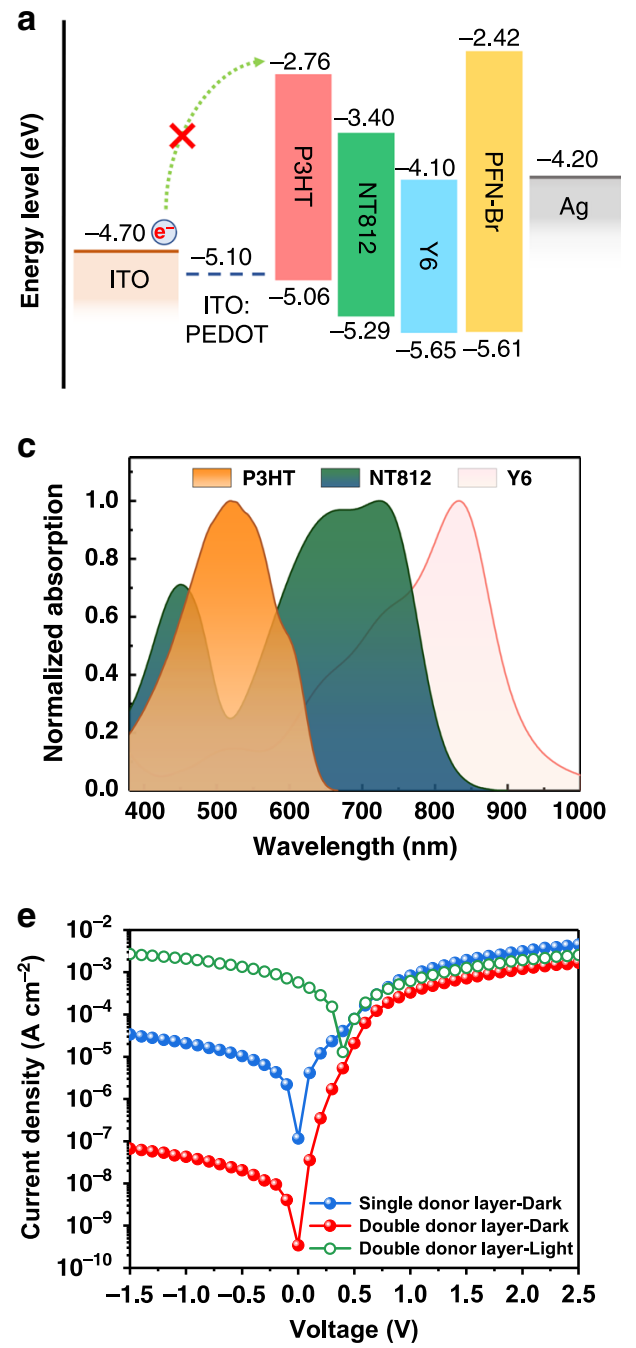

As shown in the UV-vis light absorption spectra (Fig. 3c), the absorption peak of P3HT is around $520 \mathrm{~nm}$, which just covers the dip area of the NT812 absorption spectrum. As a result, the EQE response between 500 and $600 \mathrm{~nm}$ was successfully wiped out, while the narrowband EQE peak at $860 \mathrm{~nm}$ was not affected (as shown in Fig. 3d). Here, we define two parameters, out-of-band suppression factor $S=\frac{n o r \cdot R_{\mathrm{ref}}(\lambda)}{n \text { or. } R_{\mathrm{SF}}(\lambda)}$ and in-band transmission factor $T=\frac{R_{\mathrm{SF}}\left(\lambda_{0}\right)}{R_{\mathrm{ref}}\left(\lambda_{0}\right)}$, to characterize the self-filtering property of the narrowband photodetector, where nor. $R_{\text {ref }}(\lambda)$ and nor. $R_{\mathrm{SF}}(\lambda)$ are the normalized responsivity of the reference device and SFnarrowband OPD, respectively. $R_{\text {ref }}\left(\lambda_{0}\right)$ and $R_{\mathrm{SF}}\left(\lambda_{0}\right)$ are the responsivity of the reference device and SF-narrowband OPD at the narrow respond peak, respectively. Results are shown in Supplementary Fig. 6. The maximum out-of-band suppression factor at visible region is over 170, while the in-band transmission factor at $860 \mathrm{~nm}$ is $89.2 \%$. Moreover, the spectral selectivity of our SF-narrowband OPD is based on the manipulation of excitons rather than free charge carriers after exciton dissociation ${ }^{11}$. Due to the high binding energy and the electrical neutrality of Frenkel exciton, the effect of applied electric field on the exciton diffusion is negligible ${ }^{46}$. Consequently, the EQE spectra of SF-narrowband

b
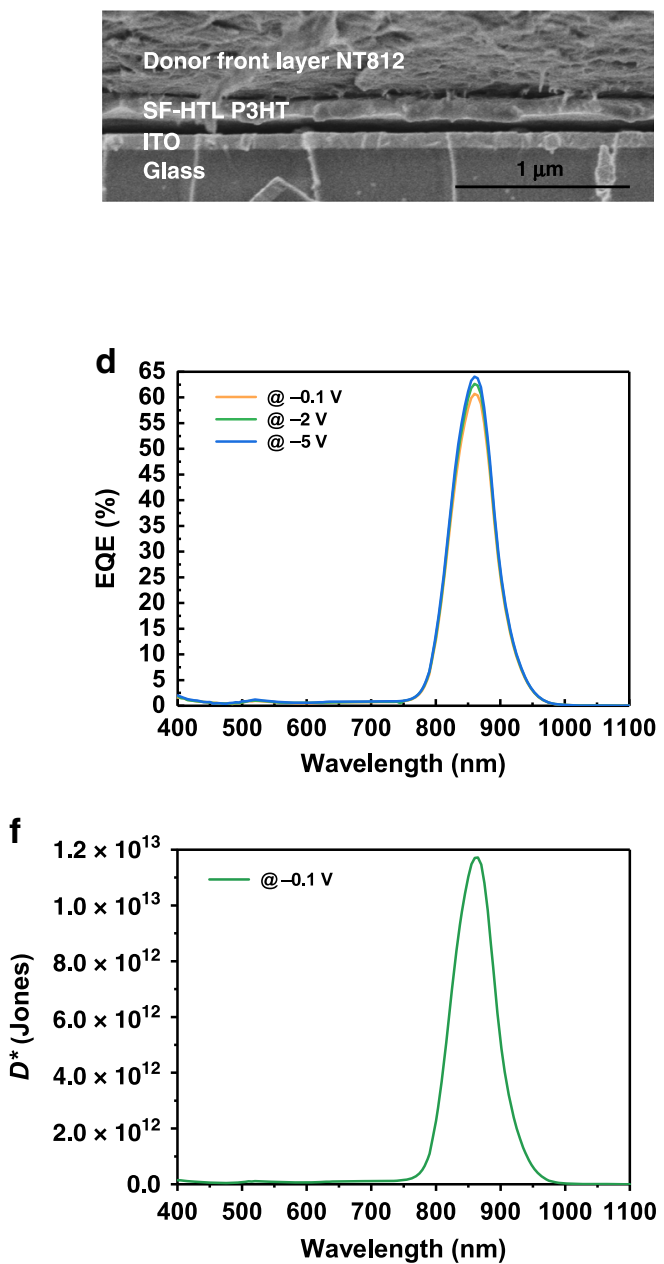

Fig. 3 Device performances of SF-narrowband OPD with double donor layers. a Energy level diagram of materials used to construct self-filtering (SF) narrowband organic photodetector (OPD) with double donor layers. b Cross-section scanning electron microscope image of the double donor layer structure, the scale bar identifies a length of $1 \mu \mathrm{m}$. c Normalized ultraviolet-visible (UV-Vis) absorption spectra of P3HT, NT812, and Y6. d External quantum efficiency (EQE) curves (at $165 \mathrm{~Hz}$ ) of the SF-narrowband OPD with double donor layers under different voltage biases as indicated. e Current density-voltage $(J-V)$ curves in dark and under illumination. $\mathbf{f}$ Specific detectivity spectra obtained from dark current density $\left(J_{d}\right)$. 
OPDs tends to saturate at higher applied voltages and retain spectral selectivity even under $-10 \mathrm{~V}$ bias (Supplementary Fig. 7), which is so significant to maintain the consistency of narrowband characteristics in practical applications.

In addition to the narrow spectral response under illumination, the dark current density $\left(J_{\mathrm{d}}\right)$ is an important feature that has a pronounced impact on specific detectivity $\left(D^{*}\right)$. As a fundamental source of electronic noise, the dark current in organic semiconductor materials is derived mainly from charge injection from the metal contacts under an applied external bias ${ }^{47}$. This injection phenomenon becomes more serious when the bandgap of the semiconductor material becomes smaller due to the reduced gaps (hence the reduced injection barrier) between the material energy levels and the electrode Fermi levels, which is one of the main factors that limits the application of narrow-bandgap organic semiconductor materials in NIR photodetectors. Figure $3 \mathrm{e}$ shows the current density-voltage $(J-V)$ characteristics of the two OPDs. Compared with the single donor layer device, the $J_{\mathrm{d}}$ of the double donor layer detector is nearly three orders of magnitude lower under reverse bias, which should be ascribed to the excellent electron blocking ability ${ }^{48}$ of wide-bandgap P3HT (Fig. 3a). Meanwhile, the cascade HOMO levels of the donor layers are favorable for the transport of photogenerated holes ${ }^{49}$, and the hole mobility of SF-HTL P3HT $(150 \mathrm{~nm}) /$ donor front layer NT812 $(750 \mathrm{~nm})$ was $1.02 \times 10^{-3} \mathrm{~cm}^{2} \mathrm{~V}^{-1} \mathrm{~s}^{-1}$ as demonstrated in Supplementary Note 2 and Supplementary Fig. 8 , so the desired EQE peak can be maintained ${ }^{50,51}$. With the incorporation of a SF-HTL P3HT, the obtained photodetectors demonstrated a peak specific detectivity $D^{*}$ of $1.2 \times 10^{13}$ Jones at $860 \mathrm{~nm}$ under $-0.1 \mathrm{~V}$ bias (as shown in Fig. 3f) calculated from the expression of $D^{*}=R / \sqrt{2 q J_{\mathrm{d}}}$, where $R$ is the responsivity (see Supplementary Fig. 9), and $q$ is the elementary charge of the electron (When the thermal noise is taken into consideration, the calculated $D^{*}$ slightly decreases to $9.5 \times 10^{12}$ Jones at $860 \mathrm{~nm}$, see the Supplementary Note 3 ). The specific detectivity curve on a logarithmic scale in Supplementary Fig. 10 shows that the specific detectivity at the detection peak is two orders of magnitude higher than that outside the detection window. The above calculation method assumes that the total noise of the photodetector is dominated by the shot noise in $J_{\mathrm{d}}$. When considered other noise, such as thermal and $1 / f$ noise, and measured the actual noise spectrum directly, a lower detectivity could be obtained according to the equation $D^{*}=R \times \sqrt{A} / S_{\mathrm{n}}$, where $A$ is the device area and $S_{\mathrm{n}}$ is the noise spectral density, yielding a peak specific detectivity $D^{*}$ of $2.4 \times 10^{12}$ Jones at $860 \mathrm{~nm}$ under a bias of $-0.1 \mathrm{~V}$ and a frequency of $165 \mathrm{~Hz}$ (see Supplementary Fig. 11). The EQE values under various illumination intensity is also a critical performance parameter for photodiodes, and the result for the double donor layer device is shown in Supplementary Fig. 12. These critical parameters, including pretty high peak EQE value, responsivity and $D^{*}$ value, make our device perform much better than previously reported no-gain type narrowband OPDs, and even comparable to commercialized silicon photodetectors (see Supplementary Fig. 13).

Universality of self-filtering narrowband OPD structure. The generic device structure proposed in this study is also applicable to other common organic semiconductor materials. The position and full-width-at-half-maximum (FWHM) of the response peaks can both be tuned by simply adjusting the combination of the donor and the acceptor materials in this study. Generally, when adopt donor and acceptor materials with red-shifted absorption onsets, the response peak will simultaneously red-shift, and when the absorption spectra of donor and acceptor materials overlap more with each other, the FWHM will be narrowed. Take the device structure based on P3HT as the SF-HTL (as shown in the Fig. 4a) for an example. The selected donor materials and acceptor materials are summarized in the Fig. $4 \mathrm{~b}$. As the normalized EQE spectra shown in the Fig. 4c, keeping the acceptor material Y6 unchanged, when the donor material NT812 was replaced by DT-PDPP2T-TT ${ }^{52}$, whose absorption onset is further redshifted than NT812 (Supplementary Fig. 14a), the FWHM of response peak was compressed from 72 to $43 \mathrm{~nm}$, and the peak position was also red shifted from 860 to $910 \mathrm{~nm}$. Furthermore, based on this donor front layer, when the acceptor material was changed from $\mathrm{Y} 6$ to IEICO- $4 \mathrm{~F}^{53}$, whose absorption onset is further redshifted than Y6 (Supplementary Fig. 14b), the response peak was further red shifted to $940 \mathrm{~nm}$ with a FWHM of $66 \mathrm{~nm}$. And as shown in Fig. 4d, both photodetectors exhibit extremely low dark current density, delivering a peak specific detectivity $D^{*}$ about $10^{13}$ Jones in their respective detection windows (as shown in Fig. 4e, f, corresponding responsivity are shown in Supplementary Fig. 15), demonstrating the good universality of this EDN approach.

\section{Discussion}

In conclusion, we successfully constructed narrowband OPDs by addressing the disadvantages of a large binding energy and the low diffusion length of photogenerated Frenkel excitons in organic semiconductors. By calculating the diffusion length and hence the distribution of the photogenerated excitons in the donor layer, we could intentionally manipulate the dissociation efficiency of excitons generated by various wavelengths of incident light via a hierarchical device structure. The SF-narrowband OPDs were thus achieved via the concept of EDN. This strategy avoids the undesirable sensitivity degradation accompanied with the thick $\mathrm{BHJ}$. Compared with conventional thin junction device under the same operating voltage, as the response in the visible range is completely suppressed, the peak EQE in the narrow NIR detection window still retains more than $89 \%$ of the corresponding value. Moreover, the utilizing of Frenkel exciton endows the resulting detector with electrically stable spectral selectivity, thus the device can still maintain narrow response even at $-10 \mathrm{~V}$ bias, achieving a peak EQE value around $65 \%$. In addition, the multilayer structure improved the charge injection barrier and effectively suppressed dark current, leading to higher detectivity. As consequence, a series of visible-blind NIR narrowband OPDs with FWHM of around $50 \mathrm{~nm}$, peak detectivity over $10^{13}$ Jones were demonstrated. This novel device structure along with its generic design concept may endow organic semiconductors with the ability to create filter-free narrowband OPDs for practical applications, examples include active imaging for face identification in smart phones, for augmented/virtual reality goggles and for full-weather robots. The narrow wavelength selectivity at 860,910 , and $940 \mathrm{~nm}$ and the corresponding performance parameters demonstrated in this work provide an "ideal" photodetector for artificial intelligent applications. It should be noted that, different from the response peak which is highly dependent on the absorption spectra of donor and acceptor, the EQE and $D^{*}$ are affected by the photon-electron conversion efficiency of donor and acceptor combination. Therefore, narrowband OPDs with desired response peak, high EQE and $D^{*}$ can be expected by selecting existing donor and acceptor materials, or designing new materials (needs to co-work with material scientist).

\section{Methods}

Materials. P3HT, DT-PDPP2T-TT, IEICO-4F, Y6, and all reagents were purchased from commercial sources (1-Material and Solarmer Materials Inc.) and used without further purification. The polymer NT812 and the crosslinker s-4FPA were synthesized in-house following established procedures in the refs. ${ }^{30,37}$. 

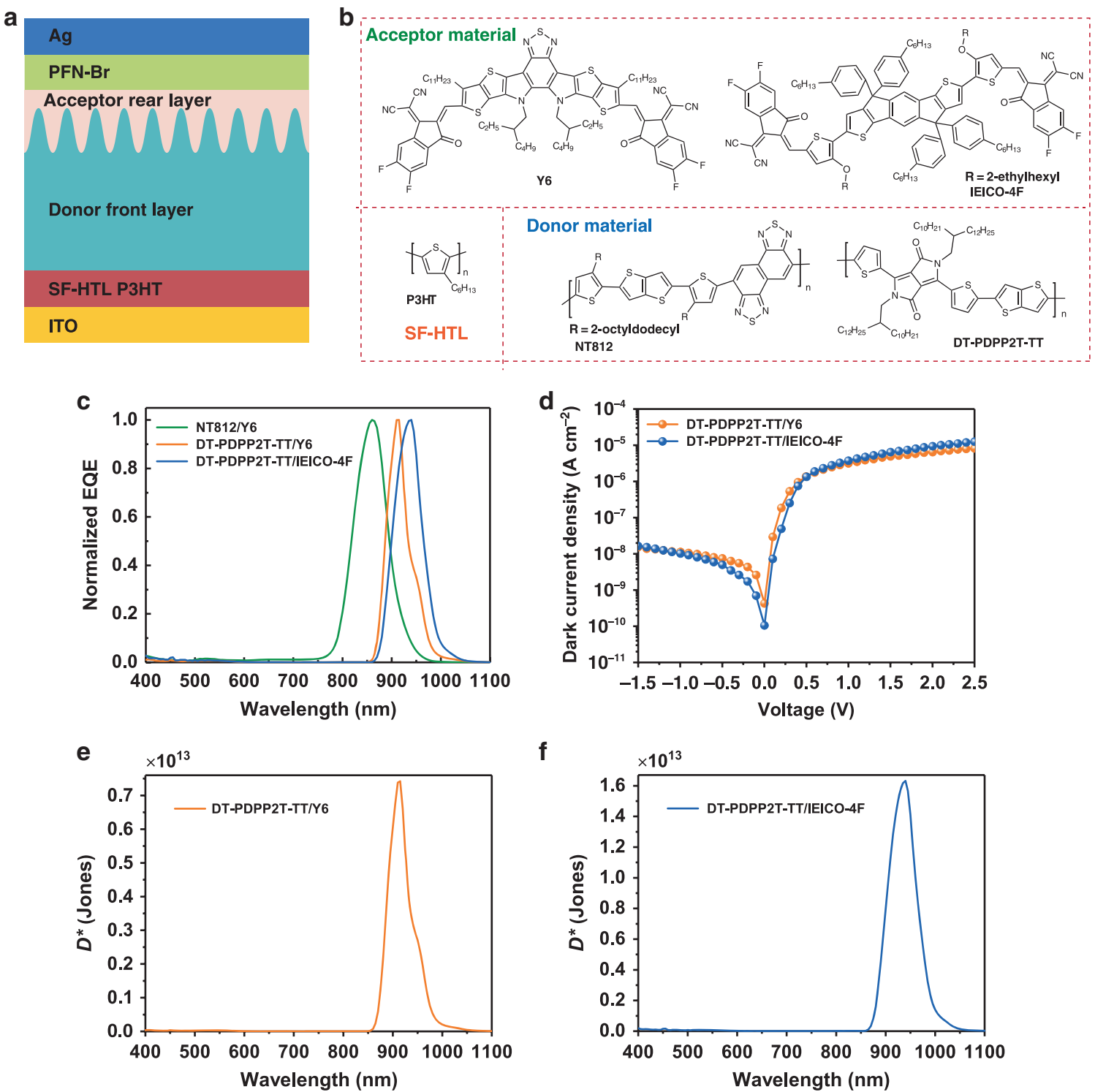

Fig. 4 Universality of the SF-narrowband OPD. a Typical device structure of self-filtering (SF) narrowband organic photodetector (OPD) with double donor layers. b Chemical structures of the selected donor materials, acceptor materials and SF hole transport layer (SF-HTL) materials. c Normalized external quantum efficiency (EQE) spectra of SF-narrowband OPDs fabricated by matching different materials, all devices were measured at $-0.1 \mathrm{~V}$ bias. d Dark current density of SF-narrowband OPDs based on DT-PDPP2T-TT/Y6 and DT-PDPP2T-TT/IEICO-4F. e Specific detectivity spectra of OPDs based on DT-PDPP2T-TT/Y6 and I DT-PDPP2T-TT/IEICO-4F, respectively.

Device fabrication. ITO-coated glass was used as the substrate. Before device fabrication, the substrates were thoroughly cleaned by sequentially sonication with acetone, isopropanol, detergent, de-ionized water, isopropanol, and subsequently dried in a baking oven over night. After that, the substrates were treated by oxygen plasma for $4 \mathrm{~min}$. For single donor layer device, PEDOT:PSS (P VP Al 4083) of $30 \mathrm{~nm}$ was first spun onto the substrates and then annealed at $150^{\circ} \mathrm{C}$ on a hot plate for $20 \mathrm{~min}$ in air to remove the residual water. NT812 mixed with $10 \mathrm{wt} \% \mathrm{~s}-4 \mathrm{FPA}$ was dissolved in CB solvent, the blend of NT812 and s-4FPA was spun onto the PEDOT:PSS, annealed at $90^{\circ} \mathrm{C}$ for $5 \mathrm{~min}$ in the glove box with nitrogen atmosphere, photo-exposed to deep-ultraviolet light (DUV, $254 \mathrm{~nm}$ wavelength), a crosslinked film of about $650 \mathrm{~nm}$ was then obtained. NT812 was dissolved in CB solution, and then spun onto the crosslinked film to obtain a thin film of about $100 \mathrm{~nm}$. Y6 was dissolved in CF solvent, and then spun onto the donor front layer to obtain a complete activelayer with a total thickness of $800 \mathrm{~nm}$. After spin-coating of $8 \mathrm{~nm}$ PFN-Br as cathode interface, a $100 \mathrm{~nm}$ Ag layer was sequentially deposited by thermal evaporation through a shadow mask in a vacuum chamber at a pressure of $4 \times 10^{-7}$ torr. For double donor layer devices based on NT812/Y6, P3HT mixed with $12 \mathrm{wt} \% \mathrm{~s}-4 \mathrm{FPA}$ was dissolved in CB solvent, the blend of P3HT and s-4FPA was spun onto the ITO substrates, annealed at $90^{\circ} \mathrm{C}$ for $5 \mathrm{~min}$ in the glove box with nitrogen atmosphere, photo-exposed to deep-ultraviolet light (DUV, $254 \mathrm{~nm}$ wavelength), and then the film was developed with CF to obtain the SF-HTL with a thickness of $150 \mathrm{~nm}$. The following fabrication steps were the same as in single donor layer devices. For double donor layer devices based on DT-PDPP2T-TT/Y6, DT-PDPP2T-T mixed with $10 \mathrm{wt} \% \mathrm{~s}-4 \mathrm{FPA}$ was dissolved in CF solvent, the blend of DT-PDPP2T-T and s-4FPA was spun onto the SF-HTL P3HT (200 nm), photoexposed to deep-ultraviolet light (DUV, $254 \mathrm{~nm}$ wavelength), a crosslinked DTPDPP2T-T film of about $1400 \mathrm{~nm}$ was then obtained. DT-PDPP2T-T was dissolved in CF solution, and then spun onto the crosslinked film to obtain a thin film of about $100 \mathrm{~nm}$. Y6 was dissolved in CF solvent, and then spun onto the donor front layer to obtain a complete activelayer with a total thickness of $1800 \mathrm{~nm}$. For double donor layer device based on DT-PDPP2T-TT/IEICO-4F, the fabrication methods of $200 \mathrm{~nm}$ SF-HTL P3HT/1400 nm crosslinked DT-PDPP2T-T/150 nm DT-PDPP2T-T were the same as DT-PDPP2T-TT/Y6-based device, IEICO-4F was dissolved in $\mathrm{CF}$, then spun onto the donor front layer to obtain a complete activelayer with a total thickness of $2000 \mathrm{~nm}$. The following fabrication steps were the same as in single donor layer devices. The device active area was $0.0516 \mathrm{~cm}^{2}$.

Device characterization. The thickness of the thin films was determined by a Dektak 150 surface profiler. The EQE spectrum was measured on a commercial 
measurement system DSR100UV-B (Zolix Instruments Co., Ltd.) equipped with DC module. The light intensity at each wavelength was calibrated using a standard single crystal Si photovoltaic cell before the testing. The light frequency was $165 \mathrm{~Hz}$. The light intensity was $2.4 \mu \mathrm{w} \mathrm{cm} \mathrm{cm}^{-2}$ at $860 \mathrm{~nm}, 2.8 \mu \mathrm{w} \mathrm{cm}-2$ at $910 \mathrm{~nm}$ and $3.1 \mu \mathrm{w} \mathrm{cm} \mathrm{cm}^{-2}$ at $940 \mathrm{~nm}$, respectively. The dark current density-voltage characteristics of the devices were recorded on a Keithley 2450 source-meter in an electrically and optically shielded box. The noise spectral density characteristics of the devices were recorded by a semiconductor parameter analyzer (Platform Design Automation, Inc. FS380 Pro). The frequency dependent measurements were carried out using a lightemitting diode $(860 \mathrm{~nm})$ modulated by the function generator (Aim-TTi TG120) as the excitation source. Square waves with different frequencies were applied. The photocurrent response of the photodiode was recorded using a digital storage oscilloscope (Tektronix TDS3052B).

ToF-SIMS depth profiling. A ToF-SIMS 5-100 (ION-TOF GmbH, Germany) instrument was used to acquire depth profiles from the prepared film. The instrument was equipped with a $\mathrm{Bi} / \mathrm{Mn}$ liquid metal ion gun (LMIG) and an argon gas cluster ion gun, which were operated in the dual beam mode. For depth profiling, a $2.5 \mathrm{keV}$ Ar-Cluster beam was used to sputter through the film at an area of $300 \mu \mathrm{m} \times 300 \mu \mathrm{m}$ in $5 \mathrm{~s}$ intervals. A $25 \mathrm{keV} \mathrm{Bi}{ }_{3}{ }^{+}$analysis beam was used to analyze the central area between sputtering pulses over a $100 \mu \mathrm{m} \times 100 \mu \mathrm{m}$ area inside the crater. The negative polarity data were used for sample analysis. The three-dimensional secondary ion images were reconstructed from the depth profile data.

Time-resolved photoluminescence (TRPL). The TRPL measurements were carried out by time-correlated single photon counting (TCSPC) technique: Acton SP2150i spectrometer (Princeton instrument) equipped with a photomultiplier (PMA 182-N-M) were used to collect the photons, an event timer (HydraHarp-400 TCSPC) with 2 ps time resolution was used to measure the fluorescence decays, a $670 \mathrm{~nm}$ pulse laser was generated by the $80 \mathrm{MHz}$ femtosecond laser with full width at half maximum about $120 \mathrm{fs}$, and the decay time fitting procedure was realized by the software FluoFit applying deconvolution with the instrument response function (IRF).

UV-Vis absorption and transmittance spectra. UV-Vis absorption spectra were acquired on a Shimadzu UV3600 spectrophotometer. Transmittance spectra were measured on an HP 8453E spectrophotometer.

Scanning electron microscope. The scanning electron microscope images were obtained with a Hitachi S-4800 FESEM.

\section{Data availability}

The data that support the findings of this study are available from the corresponding author upon reasonable request.

Received: 21 February 2020; Accepted: 13 May 2020;

Published online: 08 June 2020

\section{References}

1. Jansen-van Vuuren, R. D., Armin, A., Pandey, A. K., Burn, P. L. \& Meredith, P. Organic photodiodes: the future of full color detection and image sensing. Adv. Mater. 28, 4766-4802 (2016).

2. de Arquer, F. P. G., Armin, A., Meredith, P. \& Sargent, E. H. Solutionprocessed semiconductors for next-generation photodetectors. Nat. Rev. Mater. 2, 16100 (2017).

3. Johnston, M. Colour-selective photodiodes. Nat. Photon. 9, 634-636 (2015)

4. Lukac, R. Single-sensor imaging in consumer digital cameras: a survey of recent advances and future directions. J. Real. Time Image Proc. 1, 45-52 (2006).

5. Nishiwaki, S., Nakamura, T., Hiramoto, M., Fujii, T. \& Suzuki, M. Efficient colour splitters for high-pixel-density image sensors. Nat. Photon. 7, 240 (2013).

6. Jansen-van Vuuren, R. D., Pivrikas, A., Pandey, A. K. \& Burn, P. L. Colour selective organic photodetectors utilizing ketocyanine-cored dendrimers. J. Mater. Chem. C 1, 3532-3543 (2013).

7. Zhong, Y. et al. Helical nanoribbons for ultra-narrowband photodetectors. $J$. Am. Chem. Soc. 139, 5644-5647 (2017).

8. Siegmund, B. et al. Organic narrowband near-infrared photodetectors based on intermolecular charge-transfer absorption. Nat. Commun. 8, 15421 (2017).

9. Tang, Z. et al. Polymer:fullerene bimolecular crystals for near-infrared spectroscopic photodetectors. Adv. Mater. 29, 1702184 (2017).
10. Sobhani, A. et al. Narrowband photodetection in the near-infrared with a plasmon-induced hot electron device. Nat. Commun. 4, 1643 (2013).

11. Armin, A., Jansen-van Vuuren, R. D., Kopidakis, N., Burn, P. L. \& Meredith P. Narrowband light detection via internal quantum efficiency manipulation of organic photodiodes. Nat. Commun. 6, 6343 (2015).

12. Lin, Q., Armin, A., Burn, P. L. \& Meredith, P. Filterless narrowband visible photodetectors. Nat. Photon. 9, 687-694 (2015).

13. Fang, Y., Dong, Q., Shao, Y., Yuan, Y. \& Huang, J. Highly narrowband perovskite single-crystal photodetectors enabled by surface-charge recombination. Nat. Photon. 9, 679-686 (2015).

14. Xie, B., Chen, Z., Ying, L., Huang, F. \& Cao, Y. Near-infrared organic photoelectric materials for light-harvesting systems: organic photovoltaics and organic photodiodes. InfoMat 2, 57-91 (2019)

15. Baeg, K.-J., Binda, M., Natali, D., Caironi, M. \& Noh, Y.-Y. Organic light detectors: photodiodes and phototransistors. Adv. Mater. 25, 4267-4295 (2013).

16. Yu, G., Wang, J., McElvain, J. \& Heeger, A. J. Large-area, full-color image sensors made with semiconducting polymers. Adv. Mater. 10, 1431-1434 (1998).

17. Gong, X. et al. High-detectivity polymer photodetectors with spectral response from $300 \mathrm{~nm}$ to $1450 \mathrm{~nm}$. Science 325, 1665-1667 (2009).

18. Huang, J. et al. A high-performance solution-processed organic photodetector for near-infrared sensing. Adv. Mater. 32, 1906027 (2020).

19. van Duijnen, P. T., de Gier, H. D., Broer, R. \& Havenith, R. W. A. The behaviour of charge distributions in dielectric media. Chem. Phys. Lett. 615, 83-88 (2014).

20. Few, S., Frost, J. M. \& Nelson, J. Models of charge pair generation in organic solar cells. Phys. Chem. Chem. Phys. 17, 2311-2325 (2015).

21. Knupfer, M. Exciton binding energies in organic semiconductors. Appl. Phys. A 77, 623-626 (2003).

22. Menke, S. M., Luhman, W. A. \& Holmes, R. J. Tailored exciton diffusion in organic photovoltaic cells for enhanced power conversion efficiency. Nat. Mater. 12, 152-157 (2013).

23. Liu, X. et al. A high dielectric constant non-fullerene acceptor for efficient bulk-heterojunction organic solar cells. J. Mater. Chem. A 6, 395-403 (2018).

24. Torabi, S. et al. Strategy for enhancing the dielectric constant of organic semiconductors without sacrificing charge carrier mobility and solubility. Adv. Funct. Mater. 25, 150-157 (2015).

25. Donaghey, J. E., Armin, A., Burn, P. L. \& Meredith, P. Dielectric constant enhancement of non-fullerene acceptors via side-chain modification. Chem. Commun. 51, 14115-14118 (2015).

26. Grancini, G. et al. Hot exciton dissociation in polymer solar cells. Nat. Mater 12, 29-33 (2013).

27. Jailaubekov, A. E. et al. Hot charge-transfer excitons set the time limit for charge separation at donor/acceptor interfaces in organic photovoltaics. Nat. Mater. 12, 66-73 (2013).

28. Miao, J., Zhang, F., Du, M., Wang, W. \& Fang, Y. Photomultiplication type organic photodetectors with broadband and narrowband response ability. Adv. Opt. Mater. 6, 1800001 (2018).

29. Wang, W. et al. Highly narrowband photomultiplication type organic photodetectors. Nano Lett. 17, 1995-2002 (2017).

30. Jin, Y. et al. A novel naphtho[1,2-c:5,6-c']bis([1,2,5]thiadiazole)-based narrow-bandgap $\pi$-conjugated polymer with power conversion efficiency over 10\%. Adv. Mater. 28, 9811-9818 (2016).

31. Yuan, J. et al. Single-junction organic solar cell with over $15 \%$ efficiency using fused-ring acceptor with electron-deficient core. Joule 3, 1140-1151 (2019).

32. Liu, B. et al. High internal quantum efficiency in fullerene solar cells based on crosslinked polymer donor networks. Nat. Commun. 3, 1321 (2012).

33. Huang, F., Wu, H., Wang, D., Yang, W. \& Cao, Y. Novel electroluminescent conjugated polyelectrolytes based on polyfluorene. Chem. Mater. 16, 708-716 (2004).

34. Armin, A. et al. A shockley-type polymer: fullerene solar cell. Adv. Energy Mater. 8, 1701450 (2018).

35. Sun, R. et al. A multi-objective optimization-based layer-by-layer bladecoating approach for organic solar cells: rational control of vertical stratification for high performance. Energy Environ. Sci. 12, 3118-3132 (2019).

36. Zhang, J. et al. Sequentially deposited versus conventional nonfullerene organic solar cells: interfacial trap states, vertical stratification, and exciton dissociation. Adv. Energy Mater. 9, 1902145 (2019).

37. Png, R. Q. et al. High-performance polymer semiconducting heterostructure devices by nitrene-mediated photocrosslinking of alkyl side chains. Nat. Mater. 9, 152-158 (2010).

38. Shoaee, S. et al. Charge photogeneration for a series of thiazolo-thiazole donor polymers blended with the fullerene electron acceptors PCBM and ICBA. Adv. Funct. Mater. 23, 3286-3298 (2013).

39. Taylor, A. J., Graham, D. J. \& Castner, D. G. Reconstructing accurate ToFSIMS depth profiles for organic materials with differential sputter rates. Analyst 140, 6005-6014 (2015). 
40. Stübinger, T. \& Brütting, W. Exciton diffusion and optical interference in organic donor-acceptor photovoltaic cells. J. Appl. Phys. 90, 3632-3641 (2001).

41. Mikhnenko, O. V. et al. Exciton diffusion length in narrow bandgap polymers. Energy Environ. Sci. 5, 6960 (2012).

42. Groves, C., Blakesley, J. C. \& Greenham, N. C. Effect of charge trapping on geminate recombination and polymer solar cell performance. Nano Lett. 10, 1063-1069 (2010).

43. McNeill, C. R., Westenhoff, S., Groves, C., Friend, R. H. \& Greenham, N. C. Influence of nanoscale phase separation on the charge generation dynamics and photovoltaic performance of conjugated polymer blends: balancing charge generation and separation. J. Phys. Chem. C 111, 19153-19160 (2007).

44. Swinehart, D. F. The beer-lambert law. J. Chem. Educ. 39, 333 (1962).

45. Jung, E. H. et al. Efficient, stable and scalable perovskite solar cells using poly (3-hexylthiophene). Nature 567, 511-515 (2019).

46. Bassler, H. \& Kohler, A. "Hot or cold": how do charge transfer states at the donor-acceptor interface of an organic solar cell dissociate? Phys. Chem. Chem. Phys. 17, 28451-28462 (2015).

47. $\mathrm{Wu}, \mathrm{S}$. et al. High sensitivity polymer visible-near infrared photodetectors via an inverted device structure and manipulation of injection barrier height. Small 12, 3374-3380 (2016).

48. Keivanidis, P. E., Khong, S.-H., Ho, P. K. H., Greenham, N. C. \& Friend, R. H. All-solution based device engineering of multilayer polymeric photodiodes: minimizing dark current. Appl. Phys. Lett. 94, 173303 (2009).

49. Tan, Z. K. et al. Suppressing recombination in polymer photovoltaic devices via energy-level cascades. Adv. Mater. 25, 4131-4138 (2013).

50. Shoaee, S., Stolterfoht, M. \& Neher, D. The role of mobility on charge generation, recombination, and extraction in polymer-based solar cells. Adv. Energy Mater. 8, 1703355 (2018).

51. Zhong, W., Xie, R., Ying, L., Huang, F. \& Cao, Y. High performance polymer photodetectors enabled by a naphtho[1,2-c:5,6-c']bis([1,2,5]thiadiazole) based $\pi$-conjugated polymer. Acta Polym. Sin. 2, 217-222 (2018).

52. Li, W. et al. Efficient small bandgap polymer solar cells with high fill factors for $300 \mathrm{~nm}$ thick films. Adv. Mater. 25, 3182-3186 (2013).

53. Yao, H. et al. Design, synthesis, and photovoltaic characterization of a small molecular acceptor with an ultra-narrow band gap. Angew. Chem. Int. Ed. 56, 3045-3049 (2017).

\section{Acknowledgements}

This work was financially supported by the National Key Research and Development Program of China (No. 2019YFA0705900) funded by MOST, the Natural Science Foundation of China (Nos. 21520102006), and Guangdong Major Project of Basic and Applied Basic Research (No. 2019B030302007).

\section{Author contributions}

K.Z. and F.H. conceived the ideas and coordinated the work. B.X. carried out the device fabrication and characterization, performed the measurements of SCLC, UV-Vis absorption and transmittance spectra, performed Monte Carlo simulation and the calculation of exciton concentration distribution, and analyzed the data. Q.Y. conducted the TRPL measurement and analyzed the TRPL data. R.X. synthesized NT812. Z.H. synthesized s-4FPA. B.X., K.Z., G.Y., F.H. and Y.C. contributed to manuscript preparation. All authors commented on the manuscript.

\section{Competing interests}

The authors declare no competing interests.

\section{Additional information}

Supplementary information is available for this paper at https://doi.org/10.1038/s41467020-16675-x.

Correspondence and requests for materials should be addressed to K.Z. or F.H.

Peer review information Nature Communications thanks Zhengyuan $\mathrm{Xu}$ and the other, anonymous, reviewer(s) for their contribution to the peer review of this work. Peer reviewer reports are available.

Reprints and permission information is available at http://www.nature.com/reprints

Publisher's note Springer Nature remains neutral with regard to jurisdictional claims in published maps and institutional affiliations.

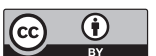

Open Access This article is licensed under a Creative Commons Attribution 4.0 International License, which permits use, sharing, adaptation, distribution and reproduction in any medium or format, as long as you give appropriate credit to the original author(s) and the source, provide a link to the Creative Commons license, and indicate if changes were made. The images or other third party material in this article are included in the article's Creative Commons license, unless indicated otherwise in a credit line to the material. If material is not included in the article's Creative Commons license and your intended use is not permitted by statutory regulation or exceeds the permitted use, you will need to obtain permission directly from the copyright holder. To view a copy of this license, visit http://creativecommons.org/ licenses/by/4.0/.

(c) The Author(s) 2020 